\title{
A note on the Hausdorff dimension of the singular set for minimizers of the Mumford-Shah energy
}

\author{
De Lellis, Camillo ; Focardi, Matteo ; Ruffini, Berardo
}

\begin{abstract}
We give a more elementary proof of a result by Ambrosio, Fusco and Hutchinson to estimate the Hausdorff dimension of the singular set of minimizers of the Mumford-Shah energy (see [Calc. Var. Partial Differential Equations 16 (2003), no. 2, 187-215, Theorem 5.6]). On the one hand, we follow the strategy of the above mentioned paper; but on the other hand our analysis greatly simplifies the argument since it relies on the compactness result proved by the first two authors in [J. Math. Pures Appl. 100 (2013), 391-409, Theorem 13] for sequences of local minimizers with vanishing gradient energy, and the regularity theory of minimal Caccioppoli partitions, rather than on the corresponding results for Almgren's area minimizing sets.
\end{abstract}

DOI: https://doi.org/10.1515/acv-2013-0107

Posted at the Zurich Open Repository and Archive, University of Zurich ZORA URL: https://doi.org/10.5167/uzh-111063

Journal Article

Published Version

Originally published at:

De Lellis, Camillo; Focardi, Matteo; Ruffini, Berardo (2014). A note on the Hausdorff dimension of the singular set for minimizers of the Mumford-Shah energy. Advances in Calculus of Variations, 7(4):539545.

DOI: https://doi.org/10.1515/acv-2013-0107 


\title{
A note on \\ the Hausdorff dimension of the singular set for minimizers of the Mumford-Shah energy
}

\author{
Camillo De Lellis, Matteo Focardi and Berardo Ruffini \\ Communicated by Nicola Fusco
}

\begin{abstract}
We give a more elementary proof of a result by Ambrosio, Fusco and Hutchinson to estimate the Hausdorff dimension of the singular set of minimizers of the MumfordShah energy (see [1, Theorem 5.6]). On the one hand, we follow the strategy of the above mentioned paper; but on the other hand our analysis greatly simplifies the argument since it relies on the compactness result proved by the first two authors in [4, Theorem 13] for sequences of local minimizers with vanishing gradient energy, and the regularity theory of minimal Caccioppoli partitions, rather than on the corresponding results for Almgren's area minimizing sets.
\end{abstract}

Keywords. Local minimizer, Mumford-Shah energy, singular set.

2010 Mathematics Subject Classification. 49J45, 49Q20.

\section{Introduction}

Consider the (localized) Mumford-Shah energy on a bounded open subset $\Omega \subset \mathbb{R}^{n}$ given by

$$
\operatorname{MS}(v, A)=\int_{A}|\nabla v|^{2} d x+\mathscr{H}^{n-1}\left(S_{v} \cap A\right),
$$

for $v \in \operatorname{SBV}(\Omega)$ and $A \subseteq \Omega$ open. In what follows if $A=\Omega$, we shall drop the dependence on the set of integration. We refer to the book [2] for all the notations and preliminaries on SBV functions and the regularity theory for local minimizers of the Mumford-Shah energy giving precise references when needed.

In this note we provide a simplified proof of the following result due to Ambrosio, Fusco and Hutchinson [1, Theorem 5.6] (shown there for quasi-minimizers as well).

The third author has been partially supported by the PRIN 2010-2011 "Calculus of Variations". 
Theorem 1.1. Let $u$ be a local minimizer of the Mumford-Shah energy, i.e., any function $u \in \operatorname{SBV}(\Omega)$ with $\operatorname{MS}(u)<\infty$ and such that

$$
\operatorname{MS}(u) \leq \operatorname{MS}(w) \text { whenever }\{w \neq u\} \subset \subset \Omega .
$$

Let $\Sigma_{u} \subseteq \overline{S_{u}}$ be the set of points out of which $\overline{S_{u}}$ is locally regular, and let

$$
\Sigma_{u}^{\prime}:=\left\{x \in \Sigma_{u}: \lim _{\rho \downarrow 0} \rho^{1-n} \int_{B_{\rho}(x)}|\nabla u|^{2}=0\right\} .
$$

Then, $\operatorname{dim}_{\mathscr{H}} \Sigma_{u}^{\prime} \leq n-2$.

The main interest in establishing such an estimate on the set $\Sigma_{u}^{\prime}$, the so-called subset of triple-junctions, is related to the understanding of the Mumford-Shah conjecture (see [2, Chapter 6] for a related discussion, see also [4, Section 7]).

Indeed, Theorem 1.1, together with the higher integrability property of the approximate gradients enjoyed by minimizers as established in two dimensions by [4] and more recently in any dimension by [5], imply straightforwardly an analogous estimate on the full singular set $\Sigma_{u}$. More precisely, in view of [4, Theorem 1] and [5, Theorem 1.1] any local minimizer $u$ of the Mumford-Shah energy is such that $|\nabla u| \in L_{\text {loc }}^{p}(\Omega)$ for some $p>2$, therefore [1, Corollary 5.7] yields that

$$
\operatorname{dim}_{\mathscr{H}} \Sigma_{u} \leq \max \{n-2, n-p / 2\} .
$$

A characterization of the Mumford-Shah conjecture in two dimensions in terms of a refined higher integrability property of the gradient in the finer scale of weak Lebesgue spaces has been recently established in [4, Proposition 5].

Our proof of Theorem 1.1 rests on a compactness result proved by the first two authors (see [4, Theorem 13]) showing that the blow-up limits of the jump set $S_{u}$ in points in the regime of small gradients, i.e., in points of $\Sigma_{u}^{\prime}$, are minimal Caccioppoli partitions. The original approach in [1], instead, relies on the notion of Almgren's area minimizing sets, for which an interesting but technically demanding analysis of the composition of SBV functions with Lipschitz deformations (not necessarily one-to-one) and a revision of the regularity theory for those sets are needed (cp. with [1, Sections 2, 3 and 4]).

Given [4, Theorem 13], the regularity theory of minimal Caccioppoli partitions developed in $[7,8,10]$ and standard arguments in geometric measure theory yield the conclusion, thus bypassing the above mentioned technical complications.

We describe briefly the plan of the note: in Section 2 we introduce a few necessary definitions and recall some well-known facts about Caccioppoli partitions. In Section 3 we prove our main result and comment on some related improvements in a final remark. 


\section{Caccioppoli partitions}

In what follows $\Omega \subset \mathbb{R}^{n}$ will denote a bounded open set.

Definition 2.1. A Caccioppoli partition of $\Omega$ is a countable partition $\mathscr{E}=\left\{E_{i}\right\}_{i=1}^{\infty}$ of $\Omega$ in sets of (positive Lebesgue measure and) finite perimeter with

$$
\sum_{i=1}^{\infty} \operatorname{Per}\left(E_{i}, \Omega\right)<\infty
$$

For each Caccioppoli partition $\mathscr{E}$ we define its set of interfaces as

$$
J_{\mathscr{E}}:=\bigcup_{i \in \mathbb{N}} \partial^{*} E_{i} .
$$

The partition $\mathscr{E}$ is said to be minimal if

$$
\mathscr{H}^{n-1}\left(J_{\mathscr{E}}\right) \leq \mathscr{H}^{n-1}\left(J_{\mathscr{F}}\right)
$$

for all Caccioppoli partitions $\mathscr{F}$ for which there exists an open subset $\Omega^{\prime} \subset \subset \Omega$ with

$$
\sum_{i=1}^{\infty} \mathscr{L}^{n}\left(\left(F_{i} \triangle E_{i}\right) \cap\left(\Omega \backslash \Omega^{\prime}\right)\right)=0 .
$$

Definition 2.2. Given a Caccioppoli partition $\mathscr{E}$, we define its singular set $\Sigma_{\mathscr{E}}$ as the set of points for which the approximate tangent plane to $J_{\mathscr{E}}$ does not exist.

A characterization of the singular set $\Sigma_{\mathscr{E}}$ for minimal Caccioppoli partitions in the spirit of $\varepsilon$-regularity results is provided in the following statement (cp. with [7, Corollary 4.2.4] and [9, Theorem III.6.5]).

Theorem 2.3. Let $\Omega$ be an open set and $\mathscr{E}=\left\{E_{i}\right\}_{i \in \mathbb{N}}$ be a minimal Caccioppoli partition of $\Omega$. Then, there exists a dimensional constant $\varepsilon=\varepsilon(n)>0$ such that

$$
\Sigma_{\mathscr{E}}=\left\{x \in \Omega \cap \overline{J_{\mathscr{E}}}: \inf _{B_{\rho}(x) \subset \subset \Omega} e(x, \rho) \geq \varepsilon\right\},
$$

where $e(x, \rho)$ denotes the spherical excess of $\mathscr{E}$ at the point $x \in J_{\mathscr{E}}$ at the scale $\rho>0$, that is,

$$
e(x, \rho):=\min _{\nu \in \mathbb{S}^{n-1}} \frac{1}{\rho^{n-1}} \int_{B_{\rho}(x) \cap J_{\mathscr{E}}} \frac{\left|v_{\mathscr{E}}(y)-v\right|^{2}}{2} d \mathscr{H}^{n-1}(y) .
$$

We recall next a result that is probably well known in literature; we provide the proof for the sake of completeness. 
Theorem 2.4. Let $\mathscr{E}$ be a minimal Caccioppoli partition in $\Omega$. Then

$$
\operatorname{dim}_{\mathscr{H}} \Sigma_{\mathscr{E}} \leq n-2 .
$$

If, in addition, $n=2$, then $\Sigma_{\mathscr{E}}$ is locally finite.

Proof. We apply the abstract version of Federer's reduction argument in [13, Theorem A.4] with the set of functions

$$
\mathcal{F}=\left\{\chi_{J_{\mathscr{E}}}: \mathscr{E} \text { is a minimal Caccioppoli partition }\right\}
$$

endowed with the convergence

$$
\chi_{J_{\mathscr{E}_{h}}} \rightarrow \chi_{J_{\mathscr{E}}} \Longleftrightarrow \lim _{h \uparrow \infty} \int_{J_{\mathscr{E}_{h}}} g d \mathscr{H}^{n-1}=\int_{J_{\mathscr{E}}} g d \mathscr{H}^{n-1} \quad \text { for all } g \in C_{c}^{1}(\Omega),
$$

and singularity map $\operatorname{sing}\left(\chi_{\mathscr{E}}\right)=\Sigma_{\mathscr{E}}$.

It is easy to see that [13, condition $A .1]$ (closure under scaling) and [13, condition $A .3$ (2)] hold true. Moreover, the blow-ups of a minimal Caccioppoli partition converge to a minimizing cone (see [8, Theorem 3.5] or [7, Theorem 4.4.5 (a)]), so that [13, condition A.2] holds as well. About [13, condition A.3 (1)], we notice that the singular set of an hyperplane is empty. Eventually, if a sequence $\left(\chi_{J_{\mathscr{E}_{h}}}\right)_{h \in \mathbb{N}} \subseteq \mathcal{F}$ converges to $\chi_{J_{\mathscr{E}}}$ and $\left(x_{h}\right)_{h \in \mathbb{N}}$ converges to $x$, with $x_{h} \in \Sigma_{\mathscr{E}_{h}}$ for all $h$, then by the continuity of the excess and the characterization in (2.1), $x \in \Sigma_{\mathscr{E}}$, so that [13, condition A.3 (3)] is satisfied as well.

To conclude, we recall that [13, Theorem A.4] itself ensures that the set $\Sigma_{\mathscr{E}}$ is locally finite being in this setting $\operatorname{dim}_{\mathscr{H}} \Sigma_{\mathscr{E}}=0$.

\section{Proof of the main result}

We are now ready to prove the main result of the note following the approach exploited in [1, Theorem 5.6]. To this aim we recall that Ambrosio, Fusco and Pallara (see [2, Theorems 8.1-8.3]) characterized alternatively the singular set $\Sigma_{u}$ as follows:

$$
\Sigma_{u}=\left\{x \in \overline{S_{u}}: \liminf _{\rho \downarrow 0}(\mathscr{D}(x, \rho)+\mathscr{A}(x, \rho)) \geq \varepsilon_{0}\right\},
$$

where $\varepsilon_{0}$ is a dimensional constant, and the scaled Dirichlet energy and the scaled mean-flatness are respectively defined as

$$
\begin{aligned}
& \mathscr{D}(x, \rho):=\rho^{1-n} \int_{B_{\rho}(x)}|\nabla u|^{2} d y, \\
& \mathscr{A}(x, \rho):=\rho^{-1-n} \min _{T \in \Pi} \int_{S_{u} \cap B_{\rho}(x)} \operatorname{dist}^{2}(y, T) d \mathscr{H}^{n-1}(y),
\end{aligned}
$$

with $\Pi$ the set of all affine $(n-1)$-planes in $\mathbb{R}^{n}$. 
Proof of Theorem 1.1. We argue by contradiction: suppose that there exists an $s>n-2$ such that $\mathscr{H}^{s}\left(\Sigma_{u}^{\prime}\right)>0$. From this we infer that $\mathscr{H}_{\infty}^{s}\left(\Sigma_{u}^{\prime}\right)>0$, and moreover that for $\mathscr{H}^{s}$-a.e. $x \in \Sigma_{u}^{\prime}$ it holds

$$
\limsup _{\rho \downarrow 0^{+}} \frac{\mathscr{H}_{\infty}^{s}\left(\Sigma_{u}^{\prime} \cap B_{\rho}(x)\right)}{\rho^{s}} \geq \frac{\omega_{s}}{2^{s}}
$$

(see for instance [2, Theorem 2.56 and formula (2.43)] or [9, Lemma III.8.15]). Without loss of generality, suppose that (3.2) holds at $x=0$, and consider a sequence $\rho_{h} \downarrow 0$ for which

$$
\mathcal{H}_{\infty}^{s}\left(\Sigma_{u}^{\prime} \cap B_{\rho_{h}}\right) \geq \frac{\omega_{s}}{2^{s+1}} \rho_{h}^{s} \quad \text { for all } h \in \mathbb{N} .
$$

Then [4, Theorem 13] provides a subsequence, not relabeled for convenience, and a minimal Caccioppoli partition $\mathscr{E}$ such that

$$
\mathscr{H}^{n-1}\left\llcorner\rho _ { h } ^ { - 1 } S _ { u } \stackrel { * } { \rightarrow } \mathscr { H } ^ { n - 1 } \left\llcorner J_{\mathscr{E}} \text { and } \rho_{h}^{-1} \overline{S_{u}} \rightarrow \overline{J_{\mathscr{E}}}\right.\right. \text { locally Hausdorff. }
$$

In turn, from the latter we claim that if $\mathscr{F}$ is any open cover of $\Sigma_{\mathscr{E}} \cap \bar{B}_{1}$, then for some $h_{0} \in \mathbb{N}$

$$
\rho_{h}^{-1} \Sigma_{u}^{\prime} \cap \bar{B}_{1} \subseteq \bigcup_{F \in \mathscr{F}} F \quad \text { for all } h \geq h_{0} .
$$

Indeed, if this is not the case we can find a sequence $x_{h_{j}} \in \rho_{h_{j}}^{-1} \Sigma_{u}^{\prime} \cap \bar{B}_{1}$ converging to some point $x_{0} \notin \Sigma_{\mathscr{E}}$. If $\pi_{x_{0}}^{\mathscr{E}}$ is the approximate tangent plane to $J_{\mathscr{E}}$ at $x_{0}$ (which exists by the very definition of $\Sigma_{\mathscr{E}}$ ), then for some $\rho_{0}$ we have

$$
\rho^{1-n} \int_{B_{\rho}\left(x_{0}\right) \cap J_{\mathscr{E}}} \operatorname{dist}^{2}\left(y, \pi_{x_{0}}^{\mathscr{E}}\right) d \mathscr{H}^{n-1}<\varepsilon_{0} \quad \text { for all } \rho \in\left(0, \rho_{0}\right) .
$$

In turn, from the latter inequality it follows that, for $\rho \in\left(0, \rho_{0} \wedge 1\right)$,

$$
\limsup _{j \uparrow \infty} \int_{B_{\rho}\left(x_{h_{j}}\right) \cap \rho_{h_{j}}^{-1} S_{u}} \operatorname{dist}^{2}\left(y, \pi_{x_{0}}^{\mathscr{E}}\right) d \mathscr{H}^{n-1}<\varepsilon_{0} .
$$

Therefore, as $x_{h_{j}} \in \rho_{h_{j}}^{-1} \Sigma_{u}^{\prime}$, we get for $j$ large enough

$$
\underset{\rho \downarrow 0}{\limsup }\left(\mathscr{D}\left(x_{h_{j}}, \rho\right)+\mathscr{A}\left(x_{h_{j}}, \rho\right)\right)<\varepsilon_{0},
$$

a contradiction in view of the characterization of the singular set in (3.1).

To conclude, we note that by (3.5) we get

$$
\mathscr{H}_{\infty}^{s}\left(\Sigma_{\mathscr{E}} \cap \overline{B_{1}}\right) \geq \limsup _{h \uparrow \infty} \mathscr{H}_{\infty}^{s}\left(\rho_{h}^{-1} \Sigma_{u}^{\prime} \cap \overline{B_{1}}\right) ;
$$


given this, (3.3) and (3.4) yield that

$$
\mathscr{H}^{s}\left(\Sigma_{\mathscr{E}} \cap \overline{B_{1}}\right) \geq \mathscr{H}_{\infty}^{s}\left(\Sigma_{\mathscr{E}} \cap \overline{B_{1}}\right) \geq \limsup _{h \uparrow \infty} \mathscr{H}_{\infty}^{s}\left(\rho_{h}^{-1} \Sigma_{u}^{\prime} \cap \overline{B_{1}}\right) \geq \frac{\omega_{s}}{2^{s+1}},
$$

thus contradicting Theorem 2.4.

Remark 3.1. In two dimensions we can actually prove that the set $\Sigma_{u}^{\prime}$ of triplejunctions is at most countable building upon some topological arguments. This claim follows straightforwardly from the compactness result [4, Theorem 13], David's $\varepsilon$-regularity theorem [3, Proposition 60.1], and a direct application of Moore's triod theorem showing that in the plane every system of disjoint triods, i.e., unions of three Jordan arcs that have all one endpoint in common and otherwise disjoint, is at most countable (see [11, Theorem 1] and [12, Proposition 2.18]). Despite this, we are not able to infer that $\Sigma_{u}^{\prime}$ is locally finite as in the case of minimal Caccioppoli partitions (cp. with Theorem 2.4). Indeed, on the one hand we can conclude that every convergent sequence $\left(x_{j}\right)_{j \in \mathbb{N}} \subset \Sigma_{u}^{\prime}$ has a limit $x_{0} \notin \Sigma_{u}^{\prime}$ thanks to [4, Proposition 11 and Lemma 12]; but on the other hand, we cannot exclude that the limit point $x_{0}$ is a crack-tip, i.e., it belongs to the set $\Sigma_{u} \backslash \Sigma_{u}^{\prime}=\left\{x \in \Sigma_{u}: \liminf _{\rho \downarrow 0} \mathscr{D}(x, \rho)>0\right\}$.

The same considerations above apply in three dimensions as well for points whose blow-up is a $\mathbb{T}$ cone, i.e., a cone with vertex the origin constructed upon the 1-skeleton of a regular tetrahedron. The latter claim follows thanks to [4, Theorem 13], the 3-d extension of David's $\varepsilon$-regularity result by Lemenant in [6, Theorem 8], and a suitable extension of Moore's theorem on triods established by Young in [15].

Let us finally point out that we employ topological arguments to compensate for monotonicity formulas, that would allow us to exploit Almgren's stratification type results and get, actually, a more precise picture of the set $\Sigma_{u}^{\prime}$ (cp. with [14, Theorem 3.2]).

\section{Bibliography}

[1] L. Ambrosio, N. Fusco and J.E. Hutchinson, Higher integrability of the gradient and dimension of the singular set for minimisers of the Mumford-Shah functionals, Calc. Var. Partial Differential Equations 16 (2003), no. 2, 187-215.

[2] L. Ambrosio, N. Fusco and D. Pallara, Functions of Bounded Variation and Free Discontinuity Problems, Oxford Math. Monogr., Clarendon Press, Oxford, 2000.

[3] G. David, Singular Sets of Minimizers for the Mumford-Shah Functional, Progr. Math. 233, Birkhäuser-Verlag, Basel, 2005.

[4] C. De Lellis and M. Focardi, Higher integrability of the gradient for minimizers of the $2 d$ Mumford-Shah energy, J. Math. Pures Appl. 100 (2013), 391-409. 
[5] G. De Philippis and A. Figalli, Higher integrability for minimizers of the MumfordShah functional, preprint (2013), http: //cvgmt.sns . it/paper/2111/.

[6] A. Lemenant, Regularity of the singular set for Mumford-Shah minimizers in $\mathbb{R}^{3}$ near a minimal cone, Ann. Sc. Norm. Super. Pisa Cl. Sci. (5) 10 (2011), no. 3, 561-609.

[7] G. P. Leonardi, Optimal subdivisions of n-dimensional domains, $\mathrm{PhD}$ thesis, Università di Trento, 1998.

[8] G. P. Leonardi, Blow-up of oriented boundaries, Rend. Semin. Mat. Univ. Padova 103 (2000), 211-232.

[9] F. Maggi, Sets of Finite Perimeter and Geometric Variational Problems, Cambridge Stud. Adv. Math. 135, Cambridge University Press, Cambridge, 2012.

[10] U. Massari and I. Tamanini, Regularity properties of optimal segmentations, J. Reine Angew. Math. 420 (1991), 61-84.

[11] R. L. Moore, Concerning triods in the plane and the junction points of plane continua, Proc. Natl. Acad. Sci. USA $\overline{14(1928)}, \overline{85-88 .}$

[12] C. Pommerenke, Boundary Behaviour of Conformal Maps, Grundlehren Math. Wiss. 299, Springer-Verlag, Berlin, 1992.

[13] L. Simon, Lectures on Geometric Measure Theory, Proc. Centre Math. Anal. Aust. Natl. Univ. 3, Australian National University, Centre for Mathematical Analysis, Canberra, 1983.

[14] B. White, Stratification of minimal surfaces, mean curvature flows, and harmonic maps, J. Reine Angew. Math. 488 (1997), 1-35.

[15] G. S. Young, Jr., A generalization of Moore's theorem on simple triods, Bull. Amer. Math. Soc. 50 (1944), 714.

Received June 3, 2013; accepted September 30, 2013.

\section{Author information}

Camillo De Lellis, Universität Zürich, Winterthurerstrasse 190,

CH-8057 Zürich, Switzerland.

E-mail: camillo.delellis@math.uzh.ch

Matteo Focardi, Università di Firenze, DiMaI "U. Dini”,

V. le Morgagni 67/A, I-50134 Firenze, Italy.

E-mail: focardi@math. unifi.it

Berardo Ruffini, Scuola Normale Superiore di Pisa,

Piazza dei Cavalieri 4, I-56126 Pisa, Italy.

E-mail: berardo.ruffini@sns.it 